\title{
The influence of cultural and religious orientations on social support and its potential impact on medication adherence
}

This article was published in the following Dove Press journal:

Patient Preference and Adherence

24 April 2015

Number of times this article has been viewed

\author{
Ernieda Hatah' \\ Kien Ping Lim ${ }^{1,2}$ \\ Adliah Mohd Ali \\ Noraida Mohamed Shah' \\ Farida Islahudin' \\ 'Faculty of Pharmacy, Universiti \\ Kebangsaan Malaysia, Jalan Raja Muda \\ Abdul Aziz, ${ }^{2}$ Pharmacy Department, \\ Kuala Lumpur General Hospital, Jalan \\ Pahang, Kuala Lumpur, Malaysia
}

Purpose: Social support can positively influence patients' health outcomes through a number of mechanisms, such as increases in patients' adherence to medication. Although there have been studies on the influence of social support on medication adherence, these studies were conducted in Western settings, not in Asian settings where cultural and religious orientations may be different. The objective of this study was to assess the effects of cultural orientation and religiosity on social support and its relation to patients' medication adherence.

Methods: This was a cross-sectional study of patients with chronic diseases in two tertiary hospitals in Selangor, Malaysia. Patients who agreed to participate in the study were asked to answer questions in the following areas: 1) perceived group and higher authority cultural orientations; 2) religiosity: organizational and non-organizational religious activities, and intrinsic religiosity; 3) perceived social support; and 4) self-reported medication adherence. Patients' medication adherence was modeled using multiple logistic regressions, and only variables with a $P$-value of $<0.25$ were included in the analysis.

Results: A total of 300 patients completed the questionnaire, with the exception of 40 participants who did not complete the cultural orientation question. The mean age of the patients was 57.6 \pm 13.5 . Group cultural orientation, organizational religious activity, non-organizational religious activity, and intrinsic religiosity demonstrated significant associations with patients' perceived social support $(r=0.181, P=0.003 ; r=0.230, P<0.001 ; r=0.135, P=0.019$; and $r=0.156, P=0.007$, respectively). In the medication adherence model, only age, duration of treatment, organizational religious activity, and disease type (human immunodeficiency virus) were found to significantly influence patients' adherence to medications (adjusted odds ratio [OR] 1.05, $P=0.002$; OR 0.99, $P=0.025$; OR 1.19, $P=0.038$; and OR 9.08, $P<0.05$, respectively).

Conclusion: When examining religious practice and cultural orientation, social support was not found to have significant influence on patients' medication adherence. Only age, duration of treatment, organizational religious activity, and disease type (human immunodeficiency virus) had significant influence on patients' adherence.

Keywords: group orientation, power distance, religiosity

\section{Introduction}

Medication adherence is described as the extent to which patients' medication-taking behavior corresponds with the prescribed medication regimen. ${ }^{1}$ Good adherence to the appropriately prescribed medications is essential for patients with chronic disease to prevent the progression of illness and death. However, according to the World Health Organization, only half of patients with chronic disease adhere to their medications. ${ }^{1}$ This scenario was also observed in Malaysia. ${ }^{2}$ In the retrospective study 
among patients with hypertension, only $53.4 \%(n=348)$ demonstrated good adherence to medication. ${ }^{2}$

Medication adherence is a complex behavioral process. It is particularly important that providers recognize every potentially modifiable risk factor for poor adherence. Medication adherence can be influenced by many factors, such as patients' demographics, complexity of treatment, and disease conditions. ${ }^{3}$ In addition, psychological factors, such as patients' perceptions of, for example, the severity and susceptibility of disease, the barriers and difficulties in taking the medication, and the benefit/cost of the medication are also reported as factors that could influence patients' adherence to medications..$^{3-5}$ Other factors that could contribute to medication adherence include patients' level of health literacy, the patient-prescriber relationship, and health care system factors. ${ }^{3}$ Nevertheless, patients' social and economic factors such as social support and medications costs, as well as religious beliefs could also influence patients' medication adherence behavior. ${ }^{3,6,7}$

Social support is defined as:

An exchange of resources between two individuals perceived by the provider or the recipient to be intended to enhance the well-being of the recipient. ${ }^{8}$

Social support can positively influence patients' health outcomes through a number of mechanisms, such as increasing patient adherence to prescribed therapies and their perceptions of quality of life, decreasing levels of depressive affect, facilitating better access to and utilization of health care, and better stress buffering through improved psychological, neuroendocrine, nutritional, or immunologic functioning. ${ }^{9}$ In a meta-analysis study by Dimatteo, the provision of social support from practical, emotional, and undimensional perspectives has been demonstrated to significantly influence patients' medication adherence. ${ }^{10}$ The positive influence of social support on medication adherence was also reported in other studies. ${ }^{11-13}$ However, those studies were conducted with Western populations where cultural factors, religious orientation, and the perceived importance of social support may be different from Asian populations.

Culture is defined as:

A set of a behaviour patterns related to thoughts, manners and action, which members of society have shared and passed on to succeeding generations. ${ }^{14}$

Culture, which involves shared characteristics, such as religion, heritage, and language, distinguishes one group of people from another. ${ }^{15}$ Culture can be understood by examining values portrayed by people's behaviors based on the fundamental assumptions and beliefs of its members. Some examples of deep-rooted Asian values that differ from western values are respecting elders, a group orientation of "we more than I", harmonious relationships, and a concern for saving face and religious orientations. ${ }^{14,15}$ Since respecting others and living in harmonious relationships are important in Asian culture, how these values influence the decision making of patients with chronic disease to take medication should be explored.

Religiosity is defined as religious beliefs (intrinsic) and involvement in religious activities (extrinsic). ${ }^{16}$ Religion has the ability to empower a person to connect to a community and to a superior force, which may lead to psychological stability. ${ }^{17}$ There is growing interest in the relationship between religion and health. In a study conducted in the United States of America, frequent attendance to religious activities improved people's health behaviors, mental health, and social relationships. ${ }^{18}$ In a different study conducted in Uganda, medication adherence was found to be associated with religious practice. In this study, patients with higher religiosity scores were found to have better adherence to antiretroviral therapy.$^{19}$ Owing to the differences in cultural and religious orientations in Asia, the influence of cultural and religious orientations on patients' perceived social support and adherence to medication is unknown.

Since Malaysia is a multiracial country that has a unique compilation of Asian populations such as Malays, Chinese, Indians, and others, it is worth exploring the cultural and religious orientations of its people and their relation to social support and medication adherence. ${ }^{20}$ In Malaysia, the three major religions, such as Islam, Christianity, and Buddhism, have substantial representation and there is a high association between religions and races. Understanding the effects of cultural and religious orientations on social support and their association with medication adherence can help in understanding potential factors for poor medication adherence.

\section{Methods}

This cross-sectional study aimed to assess the influence of cultural orientation and religiosity on social support and to investigate the relationship between cultural orientation, religiosity, and social support with medication adherence among patients with chronic disease in Malaysia. Patients attending the outpatient human immunodeficiency virus, chronic kidney disease, or hypertension clinics at two tertiary hospitals located in Selangor, Malaysia were invited to participate in the study. Data collection was done in the clinic's waiting area between April and July, 2014. The study included patients aged 18 years and above who were diagnosed with human 
immunodeficiency virus, chronic kidney disease, or hypertension, under the hospitals' follow-up, and were on medication therapy for at least 12 months. Patients were excluded from the study if they had psychotic disorders, cognitive impairments, and/or significant visual or hearing impairments. Ethics approval was granted from the Research Ethics Committee of Universiti Kebangsaan Malaysia (UKM 1.5.3.5/244/ NF-009-14) and the Medical Research and Ethics Committee of Ministry of Health Malaysia (NMRR-13-1470-18873).

Patients who agreed to participate were asked to complete the survey form comprising the following sections: 1) demographic data and treatment information; 2) perceived cultural orientation related to decisions in seeking treatment and medication usage; 3) perceived religious orientation; 4) perceived social support; and 5) perceived medication adherence behavior. Demographic data, such as age, sex, race, religion, monthly income, number of medications prescribed, number of daily doses of medication (pills, injections, and inhalations), and duration of treatment were collected to evaluate their associations with social support and medication adherence. Using a 5-point Likert-type scale, ranging from strongly disagree to strongly agree, the second section asked patients to rate their agreement to two statements: "I seek medical treatment and take medications because my family or friends asked me to do so" and "I seek medical treatment and take medications only because I feel the need to follow my doctors' orders as they know what is best for me." This section was designed to evaluate the potential influences of group orientation and the tendency to follow orders from people in higher authority when seeking treatment and using medications.

Patients' perceptions of their religious orientation were evaluated using the 5-item Duke University Religious Index questionnaire developed by Koenig and Büssing. ${ }^{16}$ The questionnaire assesses patients' religious orientation on three main domains, which are participation in organizational religious activities, participation in non-organizational religious activities, and intrinsic religiosity. The questions on organizational religious activity assess patients' frequency of attending public religious activities, such as religious services or participating in other group-related religious activity. Non-organizational religious activity refers to patients' activities performed in private, such as prayer, reading scripture, and watching or listening to religious television or radio programs. Intrinsic religiosity assesses the degree of personal religious commitment or motivation. Patients were asked to rate: 1) the frequency of participation in organizational religious and non-organizational religious activities using a 6-item Likert-type scale and 2) their agreement to intrinsic religiosity items using a 5-item Likert-type scale ranging from definitely not true to definitely true of me. The domains were examined independently from each other.

Patients' perceptions of perceived social support were evaluated using the Multidimensional Scale of Perceived Social Support by Zimet et al. ${ }^{21}$ This section reviewed patients' perceived social support from three main domains: family, friends, and significant others. Each item was rated using a 7-point Likert-type scale ranging from very strongly disagree to very strongly agree. Higher scores in this section indicate higher perceptions of the importance of social support.

Patients' perceptions of their own medication adherence were assessed using the 8-item Morisky Medication Adherence Scale. ${ }^{22}$ Patients were asked to answer yes or no to statements regarding medication adherence behavior. One mark was given for each positive answer. A strict medication adherence classification applied in a previous study was also used in this study. ${ }^{23}$ Patients were considered to be adherent if they scored 8 out of 8 in this section. The survey was prepared in English and Malay. For questionnaires that were not available in the Malay language, such as the one for cultural orientation, a standard forward-backward method was used to translate the English version.

Data were analyzed using the Statistical Package for Social Science (SPSS) version 22. Using a listwise deletion method, only completed data were analyzed. Spearman's rho correlations were used to measure the relationships between cultural orientation, religiosity, social support, and medication adherence. The tested variables (eg, cultural orientation, religiosity, social support, and other confounding variables to medication adherence, such as age, sex, monthly income, duration of treatment, number of medications, and number of daily doses) were analyzed using univariate analysis. Only variables with a $P$-value $\leq 0.25$ were included in the final model of medication adherence. Number of daily doses was not included in the final model as it correlated highly with number of medications ( $r=0.895, P<0.001)$. Using multiple logistic regressions with a backward elimination method, the final model of medication adherence was analyzed and variables with a $P$-value of $<0.05$ were considered statistically significant.

\section{Results}

During the study duration, a total of 386 study-eligible patients were invited to participate. Of these, 346 patients agreed to participate and 300 completed all sections in the questionnaire, except for 40 who did not complete the 
Table I Demographic characteristics of patients $(n=300)$

\begin{tabular}{|c|c|c|c|c|}
\hline \multirow[t]{2}{*}{ Demographic characteristics } & \multirow[t]{2}{*}{ Total or mean (SD) } & \multicolumn{3}{|c|}{ Medication adherence, mean (SD) } \\
\hline & & Non-adherent $(n=2 \mid 4)$ & Adherent $(n=86)$ & $P$-value \\
\hline Age (years) & $57.6(13.5)$ & $57.5(13.8)$ & $53.6(13.0)$ & $0.025^{a}$ \\
\hline \multicolumn{5}{|l|}{ Sex, n (\%) } \\
\hline Male & 177 & $118(39.3)$ & $59(19.7)$ & $0.032^{\mathrm{b}}$ \\
\hline Female & 123 & $96(32.0)$ & $27(9.0)$ & \\
\hline \multicolumn{5}{|l|}{ Monthly income, $\mathrm{n}(\%)$} \\
\hline$\leq \mathrm{RM} 3,000$ & 249 & $178(59.3)$ & 71 (23.7) & $0.897^{\mathrm{b}}$ \\
\hline$>\mathrm{RM} 3,000$ & 51 & $36(12.0)$ & $15(5.0)$ & \\
\hline Duration of treatment (months) & $103.9(77.6)$ & $112.2(83.3)$ & $83.1(56.6)$ & $0.005^{c}$ \\
\hline Number of medications prescribed & $5.1(2.7)$ & $5.4(2.8)$ & $4.4(2.5)$ & $0.005^{\mathrm{a}}$ \\
\hline Number of daily doses of medication & $7.7(4.8)$ & $8.2(5.0)$ & $6.5(4.1)$ & $0.002^{\mathrm{a}}$ \\
\hline
\end{tabular}

Notes: aStudent's $t$-test; 'Pearson's chi-squared test; 'Mann-Whitney U-test.

questions on cultural orientation. There were 177 males and 123 females in the study. The mean age \pm standard deviation of patients recruited into the study was $57.6 \pm 13.5$ years, with a range between 20 years and 87 years. The majority of patients were less than 65 years of age $(n=210,70.0 \%)$, and $90(30.0 \%)$ patients were aged 65 years and above. There were 131 (43.7\%) Muslims, 80 (26.3\%) Buddhists, 40 (12.3\%) Christians, 14 (4.7\%) Atheists, and 13 (4.3\%) followers of other religions (see Table 1). A total of 104 (34.7\%) patients had human immunodeficiency virus, 120 (40.0\%) were hypertensive, and 76 (25.3\%) had chronic kidney disease. The mean duration of treatment was $103.9 \pm 77.6$ months (range: 12-528 months). Patients in the study received an average of five medications (range: 1-14) or about eight doses of medications per day (range: 1-31).

Only 86 patients $(28.7 \%)$ were adherent to their medication. The majority of patients $(n=214,71.3 \%)$ were nonadherent. Based on the patients' score for cultural orientation, religiosity, and social support, the majority of patients ( $n=199,66.3 \%)$ either disagreed or strongly disagreed with the item stating that they sought treatment and used medications because their family and/or friends asked them to do so. When asked whether patients sought medical treatment or took medications because they felt that they needed to follow a doctor's instructions, the majority of patients ( $\mathrm{n}=247,95 \%)$ agreed or strongly agreed with the statement (see Table 2).

The correlation calculations regarding the influence of cultural and religious orientations on social support (see Table 3) showed that social support had weak positive significant correlations with influence of family and friends in treatment seeking and medication taking, organizational religious activity, non-organizational religious activity, and intrinsic religiosity $(r=0.181, P=0.003 ; r=0.230, P<0.001$; $r=0.135, P=0.019 ; r=0.156, P=0.007$, respectively).
When medication adherence was modeled, only age, duration of treatment, organizational religious activity, and patients with human immunodeficiency virus were shown to have significant influences on patients' adherence to medications (adjusted odds ratio [OR] 1.05, $P=0.002$; OR 0.99, $P=0.025$; and OR 9.08, $P<0.05$, respectively) (see Table 4).

\section{Discussion}

The current study evaluated the influence of cultural orientation and religiosity of patients with chronic disease on social support and adherence to medication. The majority of patients did not agree that they sought treatment and used medications because of their family or friends, but did agree to do so following doctor's instructions. Although a previous study reported that decisions related to daily life among people in Malaysia were highly influenced by group orientation, similar results may not have been observed in the current study for several reasons: 1) different study populations (eg, the present study focused on patients with chronic disease and not on the general population); 2) patients were asked to state their perceptions on specific behaviors of seeking treatment and taking medication; and 3) the location of the study included only patients who lived in urban area, where people are reported to be more individualistic. ${ }^{24}$ Although there was a significant association between group orientation and perceived importance of social support $(r=0.018$, $P=0.003)$, its association with medication adherence was not significant (OR $0.89,95 \%$ confidence interval [CI] $0.69-1.14, P=0.335)$.

Many patients agreed that they sought treatment and took medication following a doctor's instructions. This result may indicate the importance of positional power in the Malaysian population. In this culture, people with low authority usually follow instructions or orders from high power authorities, 


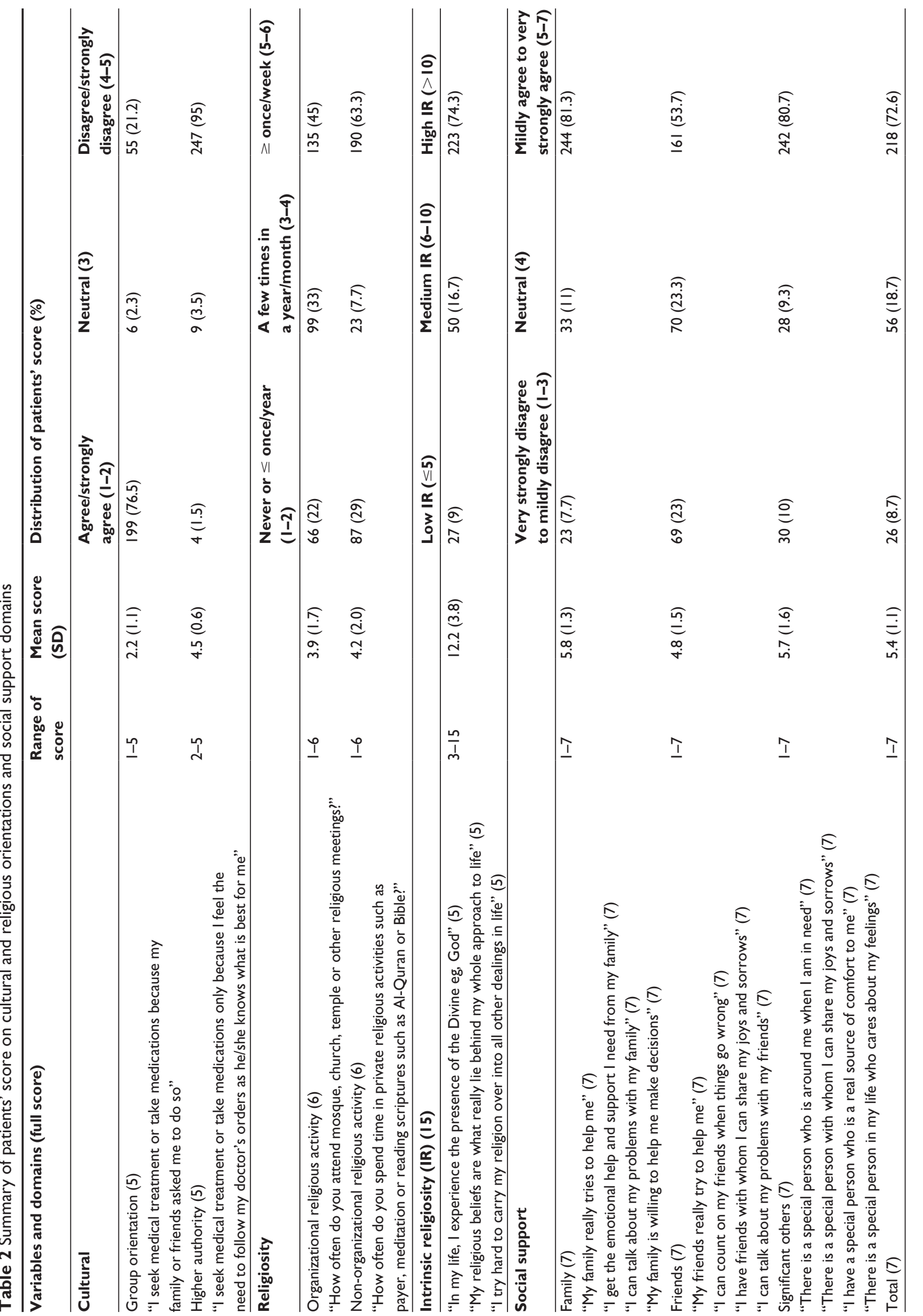


Table 3 Factors that may influence social support

\begin{tabular}{llllrr}
\hline Variable & Group orientation & Higher authority & ORA & NORA & IR \\
\hline MSPSS & & & & 0.230 & 0.135 \\
$r$ & 0.181 & -0.017 & $<0.001$ & 0.019 & 0.156 \\
$P$-value & 0.003 & 0.787 & 007 \\
\hline
\end{tabular}

Abbreviations: ORA, organizational religious activity; NORA, non-organizational religious activity; IR, intrinsic religiosity; MSPSS, multidimensional scale of perceived social support.

such as doctors, and they may not want to contradict the decisions of people in higher authority. Although the majority of patients perceived that they followed doctor's instructions when seeking treatment and using medications, the association with adherence to medications was not significant (OR $1.30,95 \%$ CI $0.83-2.04, P=1.27)$. The result may signify that patients' adherence may go beyond following instructions of people in higher authority. Following reports from previous studies, adherence to medication can be influenced by a high level of agreement between patients and doctors regarding patients' health problems and management and by the quality of the patient-doctor relationship. ${ }^{25,26}$ In these studies, medication adherence was more likely to be associated with patients who attained a higher level of patient-doctor concordance and reported that their prescribers knew them as a person. ${ }^{25,26}$

The current study found that there was no significant association between social support and medication adherence (OR $0.97,95 \%$ CI $0.90-1.04, P=0.63$ ). This finding may be due to the fact that perceived social support was measured only by general feelings, such as sharing joys and sorrows, sources of comfort, and sources with whom they could talk about their problems, which were not specific to treatment seeking and/or medication taking. A previous study has reported that among variables used to measure the influence of social support on medication adherence, practical support, such as receiving reminder to take the medications, had the highest influence on patients' adherence. In the study, the relative risk of non-adherence was found to be almost twice as high for patients who did not receive practical support than for patients who did. ${ }^{10}$

When adherence to medication was modeled, only age, organizational religious activity, duration of treatment, and patients with human immunodeficiency virus were found to be significant. Increased age was associated with an increased likelihood of medication adherence (OR 1.05, 95\% CI $1.02-1.09, P=0.002)$. A prior study on factors associated with medication adherence reported that increases in age may increase patient adherence because of greater concerns about health status. On the other hand, having other priorities

Table 4 Modeling of medication adherence

\begin{tabular}{|c|c|c|c|c|c|c|c|c|}
\hline \multirow[t]{2}{*}{ Variables } & \multicolumn{4}{|c|}{ Univariate analysis } & \multicolumn{4}{|c|}{ Multiple logistic regression } \\
\hline & Crude OR & $95 \% \mathrm{Cl}$ & $\begin{array}{l}\text { Wald's } \\
\chi^{2}(d f)\end{array}$ & $P$-value & Adjusted OR & $95 \% \mathrm{Cl}$ & $\begin{array}{l}\text { Wald's } \\
\chi^{2}(d f)\end{array}$ & $P$-value \\
\hline Age & 0.98 & $0.96-1.00$ & $4.92(I)$ & 0.027 & 1.05 & $1.02-1.08$ & $10.0(I)$ & 0.002 \\
\hline \multicolumn{9}{|l|}{ Sex } \\
\hline Male & 1.78 & $1.05-3.02$ & $4.54(\mathrm{I})$ & 0.033 & & & & \\
\hline Female & 1.00 & & & & & & & \\
\hline \multicolumn{9}{|l|}{ Monthly income } \\
\hline$\leq \mathrm{RM} 3,000$ & 0.96 & $0.49-1.86$ & $0.02(I)$ & 0.897 & & & & \\
\hline$>\mathrm{RM} 3,000$ & 1.00 & & & & & & & \\
\hline Duration of treatment & 0.99 & $0.99-1.00$ & $8.38(\mathrm{I})$ & 0.004 & 0.99 & $0.99-1.00$ & $5.01(\mathrm{I})$ & 0.025 \\
\hline Number of medications prescribed & 0.87 & $0.79-0.96$ & $7.78(1)$ & 0.005 & & & & \\
\hline Number of daily doses of medication & 0.92 & $0.87-0.97$ & $7.98(I)$ & 0.005 & & & & \\
\hline Group orientation & 0.89 & $0.69-1.14$ & $0.93(I)$ & 0.335 & & & & \\
\hline Higher authority & 1.30 & $0.83-2.04$ & $\mathrm{I} .27(\mathrm{I})$ & 0.26 & & & & \\
\hline ORA & 1.13 & $0.97-1.31$ & $2.49(1)$ & 0.115 & 1.19 & $|.06-1.4|$ & $4.30(I)$ & 0.011 \\
\hline NORA & 0.88 & $0.78-0.99$ & $4.45(I)$ & 0.035 & & & & \\
\hline IR & 0.99 & $0.93-1.06$ & $0.06(1)$ & 0.811 & & & & \\
\hline MSPSS & 0.97 & $0.90-1.04$ & $0.63(I)$ & 0.427 & & & & \\
\hline $\begin{array}{l}\text { Disease types: human immunodeficiency } \\
\text { virus (Reference to: CKD) }\end{array}$ & - & - & $33.5(2)$ & $<0.05$ & 9.08 & $3.25-25.34$ & $17.7(\mathrm{I})$ & $<0.05$ \\
\hline
\end{tabular}

Abbreviations: OR, odds ratio; $\mathrm{Cl}$, confidence interval; ORA, organizational religious activity; NORA, non-organizational religious activity; IR, intrinsic religiosity; MSPSS, multidimensional scale of perceived social support; CKD, chronic kidney disease. 
in life, such as work or commitments, may lead to lower adherence in younger patients as they may not be able to attend to their treatment. ${ }^{3}$

The current study found organizational religious activity had significant positive association with adherence to medications (OR 1.20, 95\% CI 1.01-1.14, $P=0.038$ ). It is less clear how involvement in organizational religious activity influences adherence, but one possibility may be that organizational religious activity lowers depression. ${ }^{27}$ In the study by Koenig et al patients who were more involved in organizational religious activity were found to have significant lesser depressive symptoms. ${ }^{27}$ Since depression may reduce patients' adherence to medication, lower rates of depression may be associated with better medication adherence. ${ }^{28}$ Grenard et al reported the estimated odds of a depressed patient being non-adherent were 1.76 times the odds of a non-depressed patient. ${ }^{28}$

Medication adherence was also influenced by duration of treatment (OR 0.99, 95\% CI 0.99-1.0, P=0.025). Parallel to findings from a previous study, adherence to medication was reported to reduce significantly over time. ${ }^{29}$ In the study by Cunico et al good adherence to antihyperlipidemia was observed in $90 \%$ of patients in the first 8 months of treatment, but it reduced to approximately $80 \%$ after 20 months. ${ }^{29}$ These results show that over time, it is imperative that the importance of medication adherence be emphasized for patients with long-term medication use. The current study also found that patients with human immunodeficiency virus were nine times more likely to be adherent to medication than patients with chronic kidney disease (OR 9.08, 95\% CI 3.25-25.3, $P<0.05)$. This could be because patients with HIV in the current study perceived the seriousness of their disease and the importance of adhering to their prescribed medications. A systematic review of medication adherence factors reported that disease severity, categorized based on clinical evaluation, was not associated with patients' adherence behavior. ${ }^{3}$ Medication adherence was reported to have a significant association with patients' perception of their own disease severity, rather than with disease severity based on clinical evaluation. $^{3}$

The present study has several limitations. First, the study included patients who attended follow-up clinics in hospitals; thus, it may have included patients who were more self-motivated to take care of their health. Although we may have included patients who were more motivated toward their health care, the rate of non-adherence among patients was still unsatisfactory. Second, the general measurement of social support was also a limitation. Specific measurement of social support related to medication use, such as practical support (eg, help in reminding the patients to take their medication), may have different effects on adherence to medication. Third, the small sample size that included patients with three different diseases may not represent the overall patient population in Malaysia. Finally, only using patients' self-report to evaluate medication adherence was also a limitation. Self-reporting methods may be affected by recall bias and could introduce an under- or overreported rate of medication adherence.

\section{Conclusion}

Certain aspects of cultural and religious orientations characterized in patients with chronic disease in Malaysia were found to influence the perceived importance of social support. Social support, however, was not found to have a significant influence on adherence to medication. When culture and religious orientations were evaluated, participation in organizational religious activity was shown to influence patients' medication adherence. However, there is no clear explanation of how organizational religious activity influences medication adherence behavior. Future studies might want to explore and identify how this may influence patients' medication adherence.

\section{Acknowledgments}

We would like to thank Abby Ang Shoon Yuen and Kwan Ee Wei for helping with data collection. We would also like to thank Dr Mohd Hashim Syahnaz and Dr Tong Seng Fah, associate professors of Family Medicine UKMMC; Dr Abdul Halim Abdul Gafor, associate professor and head of the Department of Nephrology UKMMC; and Dr Suresh Kumar, Infectious Disease Consultant of Hospital Sungai Buloh for their administrative support for this research.

\section{Disclosure}

The authors report no conflicts of interest in this work.

\section{References}

1. Sabaté E. Adherence to Long-Term Therapies: Evidence for Action. World Health Organization; 2003. Available from: http://whqlibdoc. who.int/publications/2003/9241545992.pdf. Accessed Nov 12, 2014.

2. Ramli A, Ahmad NS, Paraidathathu T. Medication adherence among hypertensive patients of primary health clinics in Malaysia. Patient Prefer Adherence. 2012;6:613-622.

3. Jin J, Sklar GE, Min Sen Oh V, Chuen Li S. Factors affecting therapeutic compliance: a review from the patient's perspective. Ther Clin Risk Manag. 2008;4(1):269-286.

4. Hayde JA, Paterson W. Health belief model. In: Geraci JA, Flagg AL, editors. Introduction to Health Behavior Theory. Sadbury, MA: Jones and Bartlett Publishers; 2009:31-44. 
5. Bond GG, Aiken LS, Somerville SC. The health belief model and adolescents with insulin dependent diabetes mellitus. Health Psychol. 1992; 11:190-198.

6. Wanyama J, Castelnuovo B, Wandera B, et al. Belief in divine healing can be a barrier to antiretroviral therapy adherence in Uganda. AIDS. 2007;21(11):1486-1487.

7. Wu JR, Moser DK, Chung ML, Lennie TA. Predictors of medication adherence using a multidimensional adherence model in patients with heart failure. J Card Fail. 2008;14(7):603-614.

8. Shumaker SA, Brownell A. Toward a theory of social support: closing conceptual gaps. J Soc Issues. 1984;40(4):11-36.

9. Cohen SD, Sharma T, Acquaviva K, Peterson RA, Patel SS, Kimmel PL. Social support and chronic kidney disease: an update. Adv Chronic Kidney Dis. 2002;14:335-344.

10. Dimatteo MR. Social support and patient adherence to medical treatment: a meta-analysis. Health Psychol. 2004;23(2):207-218.

11. Wu JR, Frazier SK, Rayens MK, Lennie TA, Chung ML, Moser DK. Medication adherence, social support, and event-free survival in patients with heart failure. Health Psychol. 2013;32(6):637-646.

12. Vyavaharkar M, Moneyham L, Tavakoli A, et al. Social support, coping and medication adherence among HIV-positive women with depression living in rural areas of the Southeastern United States. AIDS Patient Care STDS. 2007;21(9):667-680.

13. Hanif $\mathrm{H}$, Bastos FI, Malta M, et al. Individual and contextual factors of influence on adherence to antiretrovirals among people attending public clinics in Rio De Janeiro, Brazil. BMC Public Health. 2013;13: 574-584.

14. Jamal M. Type-A behaviour in a multinational organization: a study of two countries. Stress Health. 2006;23:101-109.

15. Talib MA. Cultural influences and mandated counseling in Malaysia. Asian Cult Hist. 2010;2(1):28-33.

16. Koenig HG, Büssing A. The Duke University Religion Index (Durel): a five-item measure for use in epidemological studies. Religions. 2010; 1(1):78-85.

17. Oman D, Thoresen CE. Without spirituality does critical health psychology risk fostering cultural iatrogenesis? J Health Psychol. 2003;8(2): 223-229.

18. Strawbridge WJ, Shema SJ, Cohen RD, Kaplan GA. Religious attendance increases survival by improving and maintaining good health behaviors, mental health, and social relationships. Ann Behav Med. 2001;23(1):68-74.
19. Kisenyi RN, Muliira JK, Ayebare E. Religiosity and adherence to antiretroviral therapy among patients attending a public hospital-based HIV/AIDS clinic in Uganda. J Relig Health. 2013;52(1):307-317.

20. Department of Statistic Malaysia. Population Distribution and Basic Demographic Characteristic 2010. 2011. Available from: http://www. statistics.gov.my/portal/index.php?option=com_content\&id=1215\&Ite mid=89\&lang=en. Accessed Nov 12, 2014.

21. Zimet GD, Dahlem NW, Zimet SG, Farley GK. The multidimensional scale of perceived social support. J Pers Assess. 1988;52(1):30-41.

22. Morisky DE, Ang A, Krousel-Wood M, Ward HJ. Predictive validity of a medication adherence measure in an outpatient setting. J Clin Hypertens (Greenwich). 2008;10(5):348-354.

23. Oliveira-Filho AD, Barreto-Filho JA, Neves SJF, Lyra JDP. Association between the 8-item Morisky Medication Adherence Scale (MMAS-8) and blood pressure control. Arq Bras Cardiol. 2012;99(1):649-658.

24. Yoko Sumikawa T, Yoshihiko Y. Relationships among sense of coherence, resources, and mental health in urban and rural residents in Japan. BMC Public Health. 2012;12:1107-1115.

25. Kerse N, Buetow S, Mainous AG, Young G, Coster G, Arroll B. Physicianpatient relationship and medication compliance: a primary care investigation. Ann Fam Med. 2004;2(5):455-461.

26. Beach MC, Keruly J, Moore RD. Is the quality of the patient-provider relationship associated with better adherence and health outcomes for patients with HIV? J Gen Intern Med. 2006;21(6):661-665.

27. Koenig HG, George LK, Titus P. Religion, spirituality, and health in medically ill hospitalized older patients. J Am Geriatr Soc. 2004;52(4): 554-562.

28. Grenard JL, Munjas BA, Adams JL, Suuorp M, McGlynn EA, Gellad WF. Depression and medication adherence in the treatment of chronic diseases in the United States: a meta-analysis. J Gen Intern Med. 2011; 26(10):1175-1182.

29. Cunico C, Picheth G, Correr CJ, Scartezini M. Assessing the adherence to and the therapeutic effectiveness of hypolipidemic agents in a population of patients in Brazil: a retrospective cohort study. Pharm Prac (Granada). 2014;12(2):378-385.
Patient Preference and Adherence

\section{Publish your work in this journal}

Patient Preference and Adherence is an international, peer-reviewed, open access journal that focuses on the growing importance of patient preference and adherence throughout the therapeutic continuum. Patient satisfaction, acceptability, quality of life, compliance, persistence and their role in developing new therapeutic modalities and compounds to optimize

\section{Dovepress}

clinical outcomes for existing disease states are major areas of interest for the journal. This journal has been accepted for indexing on PubMed Central. The manuscript management system is completely online and includes a very quick and fair peer-review system, which is all easy to use. Visit http://www dovepress.com/testimonials.php to read real quotes from published authors. 